\title{
Relaxations of the matroid axioms I: Independence, Exchange and Circuits
}

\author{
José Alejandro Samper $\rrbracket$ \\ ${ }^{1}$ Department of Mathematics, University of Washington, Seattle, USA
}

\begin{abstract}
Motivated by a question of Duval and Reiner about higher Laplacians of simplicial complexes, we describe various relaxations of the defining axioms of matroid theory to obtain larger classes of simplicial complexes that contain pure shifted simplicial complexes. The resulting classes retain some of the matroid properties and allow us to classify matroid properties according to the relevant axioms needed to prove them. We illustrate this by discussing Tutte polynomials. Furthermore, we extend a conjecture of Stanley on $h$-vectors and provide evidence to show that the extension is better suited than matroids to study the conjecture.

Résumé. Motivé par une question de Duval et Reiner concernant les Laplaciens d'ordres supérieurs des complexes simpliciaux, nous décrivons certaines relaxations des axiomes de la théorie des matroïdes afin d'obtenir des familles plus grandes de complexes simpliciaux qui contiennent les complexes simpliciaux pures décalés. Les classes obtenues conservent certaines propriétés des matroïdes et nous permettent de classifier les propriétés des matroïdes selon les axiomes nécessaires pour les démontrer. Nous illustrons ceci à l'aide des polynômes de Tutte. De plus, nous étendons une conjecture de Stanley concernant les $h$-vecteurs et supportons l'énoncé par des preuves montrant que l'extension est mieux adaptée à la conjecture que les matroïdes.
\end{abstract}

Keywords. Matroid, Shifted Complex, Tutte polynomial, h-vectors

\section{Introduction}

Matroid independence complexes and shifted simplicial complexes are two remarkable classes of simplicial complexes that appear naturally in several areas of mathematics. Matroids play a prominent role in the study of vector configurations, linear optimization, graph theory, matching theory, intersection theory in Grassmannians and various other places. The aim of matroid theory is to provide an abstract notion of independence in mathematics. On the other hand, shifted complexes play a prominent role in the combinatorial theory of simplicial complexes. Several different operations have been defined for simplicial complexes that map a complex $\Delta$ to a shifted complex $\Delta^{s}$ in a way that preserves $f$-vectors, CohenMacaulayness and more properties.

A surprising similarity between the two classes comes from the theory of combinatorial Laplacians. Let $C .(\Delta)$ be the simplicial chain complex of a simplicial complex $\Delta$ over the real numbers. The homological

†Email: samper@math. washington. edu. This work was partially supported through research assistant positions funded by Isabella Novik's NSF Grant DMS-1361423.

1365-8050 @ 2016 Discrete Mathematics and Theoretical Computer Science (DMTCS), Nancy, France 
boundary and coboundary maps, $\partial$ and $\delta$, are adjoint maps in each degree with respect to the face basis. The $k$-Laplacian $L_{k}=\partial \delta+\delta \partial$ of $\Delta$ is a self-adjoint map and therefore, has real eigenvalues. It is natural to ask if there is some relationship between the eigenvalues of $L_{k}$ and the combinatorial properties of $\Delta$.

It was shown in a series of papers (Kook et al. (2000); Duval and Reiner (2002); Kook (2004); Duval (2005)) that the eigenvalues of the Laplacians of both matroid independence and shifted simplicial complexes are integers and satisfy a special kind of recurrence similar to the deletion-contraction recurrence of the Tutte polynomial of a matroid. The proofs of the theorem are very different for both classes. Since having integral Laplacian spectrum is a remarkably rare property, Duval and Reiner asked the following.

Question 1.1 (Reiner (2001); Duval and Reiner (2002); Duval (2014)) Is there a class of simplicial complexes that contains matroid independence and shifted simplicial complexes and explains the integral Laplacian phenomenon?

In this manuscript we will discuss the first steps in the construction of a class of simplicial complexes that will hopefully answer Question 1.1 affirmatively. The basic idea is to work with ordered simplicial complexes and relax some of the different axiomatizations of matroid theory. The goal is to enlarge the class of matroids so it contains pure shifted simplicial complexes. The goal of this note is to give a brief presentation of such extensions for the following axioms: independence, exchange and circuits. Other extensions, such as semimodular functions and greedy algorithms, will be discussed in various upcoming projects.

The relaxations give a measure of the "complicatedness" of several matroid properties according to how many of the axioms are required to obtain an analogue. We show that the relaxations of the exchange and the circuit axioms allow us to define activity theories and get an analogue of the Tutte polynomial that satisfies an ordered deletion-contraction recursion.

Another virtue of our theory is that it allows us to transfer questions about matroids to analogous questions about shifted complexes. Proving theorems for shifted complexes is easier in general and one might try to imitate techniques for shifted complexes in the context of matroid theory. We illustrate this by providing a refinement of a classical conjecture of Stanley about $h$-vectors of matroids.

Conjecture 1.2 (Stanley (1977)) The $h$-vector of a matroid is a pure $\mathcal{O}$-sequence.

This says that if $\left(h_{0}, h_{1}, \ldots, h_{d}\right)$ is the $h$-vector of a matroid, then there exists a family $\mathcal{O}$ of monomials that is closed under divisibility with exactly $h_{i}$ monomials of degree $i$ and such that every monomial in $\mathcal{O}$ divides a maximum degree monomial in $\mathcal{O}$. Such a family is called a pure multicomplex. It is just a multicomplex if the last condition is ignored.. The hard part of Stanley's conjecture is to show purity. In fact, Stanley (1977) proved that such $h$-vectors count degrees in multicomplexes using Stanley-Reisner theory and the theory of Cohen-Macaulay rings.

The conjecture is still wide open despite of the big effort put into finding a solution. Several special cases are known: cographical matroids Merino (2001), paving matroids Merino et al. (2012), cotransversal matroids Oh (2013), positroids Oh (2011), rank 3 and corank 2 matroids De Loera et al. (2012), rank $d$ matroids with $1 \leq h_{d} \leq 5$ Constantinescu et al. (2014) and the recently defined class of internally perfect matroids Dall (2015). The rank 4 case was proved in Klee and Samper (2015) by providing a combinatorial recipe to construct the multicomplex that comes from an $h$-vector decomposition. We provide an analogue of that $h$-vector decomposition for complexes satisfying the relaxations of exchange 
and independence and use it to extend the combinatorial conjecture to one about complexes satisfying both relaxations. We then prove the new conjecture for shifted complexes and speculate about the general solution. The advantage of our method is that we can induct on the number of facets which is a technique that is out of reach within the confines of matroid theory.

This note is organized as follows. In Section 2 we introduce the objects we are going to study. In Section 3 we discuss the relaxations of the axioms and mention some of their properties. In Section 4 we discuss the theory of Tutte polynomials and in Section 5 we discuss the relationship between the relaxations and Stanley's conjecture.

\section{Preliminaries}

An ordered simplicial complex is a pair $\Psi=(E, \Delta)$ where $E$ is a totally ordered finite set and $\Delta$ is a pure simplicial complex whose vertex set is a subset of $E$. We will use matroid theory terminology. Faces of $\Delta$ are called as independent sets. Facets, i.e maximal under inclusion independent sets, are called bases. The set of bases is denoted by $\mathcal{B}$. It is useful to order $\mathcal{B}$ lexicographically using the order of $E$. The smallest lexicographic basis is denoted by $B_{0}$. Minimal non-faces are called circuits. The set of circuits is denoted by $\mathcal{C}$. The rank of $\Psi$ is the size of any element of $\mathcal{B}$. We usually reserve $d$ to denote the rank of $\Psi$.

A loop of $\Psi$ is an element of $E$ that is not in any basis. A coloop is an element of $\Psi$ that is in every basis. For $A \subset E$, define the restriction $\left.\Psi\right|_{A}$ to be the pair $\left(A,\left.\Delta\right|_{A}\right)$, where $\Delta_{A}=\{I \in \Delta: I \subseteq A\}$. The deletion $\Psi \backslash\{e\}$ of an element $e$ that is not a coloop is the restriction to $E \backslash\{e\}$. The contraction $\Psi /\{e\}$ of an element $e$ that is not a loop is the complex $\left(E-\{e\}, \operatorname{Link}_{\Delta}(e)\right)$. The contraction $\Psi / I$ of an independent set $I$ is the complex that results from contracting the vertices of $I$ in any order. For an independent set $I$, let $B_{I, 0}$ be the smallest lexicographic basis of $\Psi \backslash I$. The complex $(\{e\},\{\emptyset\})$ is denoted by $\Psi_{\text {loop }}$ and the complex $(\{e\},\{\emptyset,\{e\}\})$ is denoted by $\Psi_{\text {coloop }}$.

Given a basis $B$ and an element $e \in E \backslash B$, we say that $e$ is externally active if there is a circuit $C \subset B \cup\{e\}$ such that $e$ is the smallest element of $C$. We say that $e$ is externally passive otherwise. The sets of externally active and passive elements of $B$ are denoted by $E A(B)$ and $E P(B)$ respectively. An element $b \in B$ is called internally active if $B$ is the smallest lexicographic basis that contains $B \backslash\{b\}$. Equivalently, there is no $b^{\prime}<b$ that is not in $B$ and such that $(B \backslash\{b\}) \cup\left\{b^{\prime}\right\} \in \mathcal{B}$. An element is internally passive otherwise. The sets of internally active and passive elements of $B$ are denoted by $I A(B)$ and $I P(B)$ respectively.

A broken circuit is an element of the form $C-\{c\}$, where $C \in \mathcal{C}$ and $c$ is the smallest element of $c$. The $n b c$-complex $n b c(\Psi)$ of $\Psi$ is the complex $(E, \Gamma)$, such that the bases of $n b c(M)$ are the bases of $\Psi$ that don't contain a broken circuit.

The Gale order, $<$, of an ordered complex $\Psi$ is an order on the set of basis that is given as follows: if $B$ and $B^{\prime}$ are bases with elements $b_{1}<b_{2}, \cdots<b_{d}$ and $b_{1}^{\prime}<b_{2}^{\prime}<\cdots<b_{d}^{\prime}$ respectively, then $B<B^{\prime}$ if and only if $b_{i} \leq b_{i}^{\prime}$ for every $i$. The poset $(\mathcal{B},<)$ is denoted by Gale $(\Psi)$.

An ordered complex $\Psi$ is an ordered matroid if whenever $I_{1}$ and $I_{2}$ are independent sets such that $\left|I_{1}\right|>$ $\left|I_{2}\right|$ then there is an element $i \in I_{1} \backslash I_{2}$ such that $I_{2} \cup\{i\} \in \Delta$. A simple consequence of matroid duality 
(e.g see $\mathrm{Gel}^{\prime}$ fand et al. (1987)) is that $\operatorname{Gale}(\Psi)$ has a minimum and a maximum whenever $\Psi$ is an ordered matroid. There are other axioms defining matroids. Later, we will define the relaxed version in which the order of $E$ is used in some way. In each case, the original matroid axiom is the same as the relaxed one except for the condition using the order of $E$, which should be ignored.

An ordered complex $\Psi$ is shifted if every independent $I$ and $i \in I$ satisfy the following: for every $j \in E$ smaller than $i$, the set $I \backslash\{i\} \cup\{j\}$ is independent. An equivalent formulation is that an ordered complex $\Psi$ is shifted if an only if Gale $(\Psi)$ is isomorphic to an order ideal of Young's lattice of integer partitions. It is a theorem of Klivans (2003) that a shifted complex is a matroid if and only if Gale $(\Psi)$ has a maximum.

The $f$-vector of a rank $d$ complex $\Psi$ is the vector $\left(f_{0}, f_{0}, \ldots, f_{d}\right)$ where $f_{i}$ is the number of independents of rank $i$ (or dimension $i-1$ ). Notice that $f_{0}=1$. The $h$-vector $\left(h_{0}, \ldots, h_{d}\right)$ of $\Psi$, which is sometimes more convenient when studying complexes through the lens of commutative algebra, is given by the following polynomial relation

$$
h(\Psi, x):=\sum_{j=0}^{d} h_{j} x^{j}=\sum_{j=0}^{d} f_{j-1} t^{j}(1-t)^{d-j}
$$

A shelling order of a complex $\Psi$ is an order $B_{1}, \ldots B_{k}$ of the bases such that for every $i<j$ there is $k \leq j$ and $b \in B_{j}$ such that $B_{i} \cap B_{j} \subseteq B_{k} \cap B_{j}=B_{j} \backslash\{b\}$. We say that $\Psi$ is shellable if it admits a shelling order. If $B_{1}<\cdots<B_{k}$ is a shelling order, then for every $j$ there is a unique subset $\mathcal{R}\left(B_{j}\right)$ of $B_{j}$ such that $\mathcal{R}\left(B_{j}\right) \nsubseteq B_{i}$ for any $i<j$. It turns out that

$$
h(\Psi, x)=\sum_{j=1}^{k} x^{\left|\mathcal{R}\left(B_{j}\right)\right|} .
$$

For two sets $A, B$ let $A \triangle B$ be their symmetric difference, i.e, the set $(A \backslash B) \cup(B \backslash A)$. Whenever a subset of a small set is considered we drop parethensis and commas to simplify notation. For example, the subset $\{2,4\}$ of $\{1,2,3,4,5\}$ is denoted by 24 .

\section{The relaxed axioms}

We begin by defining the three axioms that we will discuss in this manuscript. The fact that matroids satisfy these axioms is straightforward, while their verification for pure shifted complex is a simple fun exercise.

Definition 3.1 Let $\Phi=(E, \Delta)$ be an ordered simplicial complex. The following relaxations of the axioms are defined.

- Weak Independence Axiom (WIA): For every pair of independent sets $I_{1}, I_{2}$, if $\left|I_{1}\right|>\left|I_{2}\right|$ and $I_{1} \backslash I \subseteq B_{I, 0}$ for some $I \subseteq I_{1} \cap I_{2}$, then there exists $e \in I_{1} \backslash I_{2}$ such that $I_{2} \cup\{e\}$ is independent.

- Weak Exchange Axiom (WEA): For every pair $B_{1}, B_{2}$ of bases of $\Delta$, and $b_{2} \in B_{1} \backslash B_{2}$ such that $b_{1}>\max B_{2} \backslash B_{1}$, then there is a $b_{2} \in B_{2} \backslash B_{1}$ such that $\left(B_{1} \backslash\left\{b_{1}\right\}\right) \cup\left\{b_{2}\right\} \in \Delta$. 
- Weak Circuit Axiom (WCA): If $C_{1}, C_{2}$ are distinct circuits $(E, \Delta)$ and $c \in C_{1} \cap C_{2}$ such that $c<\max C_{1} \triangle C_{2}$, then there is a circuit $C_{3}$ of $(E, \Delta)$ contained in $C_{1} \cup C_{2}-c$.

In matroid theory, all the axioms produce the same class of objects, on the other hand, the relaxed axioms produce different classes. The following theorem serves as an interesting example.

Theorem 3.2 The three relaxations produce distinct classes of simplicial complexes. None of the axioms is implied by another one.

Proof: The one dimensional ordered complex $\Psi_{1}=\left(\{1,2,3,4\}, \Delta_{1}\right)$ with bases $12,13,14,34$. It satisfies WIA, WEA, but the circuits 23,24 contradicts WCA.

The complex $\Psi_{2}=\left(\{1,2,3,4\}, \Delta_{2}\right)$ with bases $14,24,23,34$. It satisfies WIA, but 14 and 23 show it does not satisfy WEA or WCA.

The complex $\Psi_{3}=\left(\{1,2,3,4\}, \Delta_{3}\right)$ with bases $12,13,23,34$ satisfies WCA, but fails WIA and WEA.

The complex $\Psi_{4}=\left(\{1,2,3,4,5\}, \Delta_{4}\right)$ with bases 13, 14, 23, 24, 25 satisfies WEA, but not WIA or WCA.

We already know that there are many examples of objects satisfying each relaxation theory, and in fact, many satisfy all of them simultaneously. In order to explore more examples, a useful tool in general is to know how to construct new complexes from old ones satisfying a given axiom. The following theorem tell us what we can do with each of the axioms, in terms of simplicial joins, restrictions, contractions, connected sums and taking skeleta.

Theorem 3.3 For each individual axiom, as listed below, we have that the following operations preserve the property of satisfying the axiom:

- WIA: simplical joins, restrictions to sets $A \subseteq E$ such that $r k(A)=\left|A \cap B_{0}\right|$, contractions, connected sums along least lex bases, and skeleta.

- WEA: simplicial joins, restrictions to initial segments and contractions.

- WCA: simplicial joins, restrictions that give pure complexes, contractions and skeleta.

One highlight of the next theorem is the following: we know that matroid independence complexes are characterized as simplicial complexes all of whose restrictions are pure. This can't possibly be true for other classes of complexes, but the WIA guarantees that there are still plenty of pure induced subcomplexes. We now present a few simple consequences of each of the axioms. For each of the theorems mentioned below, the proofs are either straightforward, or careful modifications of the analoguous theorem found in Björner (1992).

Theorem 3.4 Let $\Psi=(E, \Delta)$ be a complex satisfying WIA. Then $\Delta$ is vertex decomposable and any vertex that does not belong to $B_{0}$ is a shedding vertex. This implies that $\Delta$ is shellable.

Theorem 3.5 Let $\Psi=(E, \Delta)$ be a complex satisfying WEA. Then $\Delta$ is vertex decomposable and the largest vertex is a shedding vertex. In particular $\Delta$ is shellable. The lexicographic order of the facets is a 
shelling order and for every facet $B$ the set $I P(B)$ is the unique minimal subset of $B$ not contained in a lexicographically smaller basis. Therefore we have that

$$
h(\Psi, x)=\sum_{B \in \mathcal{B}} x^{|I P(B)|} .
$$

Theorem 3.6 Let $\Psi=(E, \Delta)$ be a complex satisfying WCA. If $B$ is a facet and $e \in E-B$ is externally active, then there is a unique circuit contained in $B \cup\{e\}$.

In particular, each of the relaxations of the axioms resembles a part of matroid theory. While each of the relaxations is interesting, the real power of the theory shows up when we start considering combinations of the axioms. This gives a hierarchy for the 'complicatedness' of matroid properties, i.e, we can classify several matroid properties according to the relevant axioms that are required to prove analogues in the weak setting.

For the rest of the manuscript we focus on two aspects of matroid theory that admit interesting generalizations to the weak setting: Tutte polynomials and the structure of $h$-vectors.

\section{Tutte polynomials}

Let $\mathcal{S}$ denote the class of complexes satisfying WCA and WEA and $R$ be a commutative ring with unity. An invariant $f$ is a map that associates to every complex $\Psi$ of $\mathcal{S}$ an element $f(\Psi) \in R$. A TG-invariant is an invariant that satisfies the following recurrence:

$$
f(M)=\left\{\begin{array}{l}
f\left(\left.\Psi\right|_{\{e\}}\right) f\left(\left.\Psi\right|_{E \backslash\{e\}}\right) \text { if } e \text { is a loop or a coloop, } \\
f(\Psi / e)+f(\Psi \backslash e) \text { if } e \text { is the largest vertex that is not a coloop. }
\end{array}\right.
$$

There are several natural TG-invariants. For example, the number of faces, bases, the reverse $f$-polynomial and reverse $h$-polynomials are TG-invariants. Furthermore, for every $\Psi$ in $\mathcal{S}$, the underlying complex of $n b c(\Psi)$ is shellable and the polynomial $t^{d} h\left(n b c(\Psi), \frac{1}{t}\right)$ is a TG-invariant. It turns out that there is a polynomial that governs all TG-invariants.

Definition 4.1 Let $\Psi$ be a complex in $\mathcal{S}$. The Tutte polynomial of $\Psi$ is defined to be

$$
T(\Psi, x, y):=\sum_{B \in \mathcal{B}} x^{|I A(B)|} y^{|E A(B)|} .
$$

Theorem 4.2 The Tutte polynomial is a TG-invariant that is universal in the following sense: if $f$ is a TG-invariant in the class $\mathcal{S}$, then for every $\Psi$ in $\mathcal{S}$ we have that

$$
f(\Psi)=T\left(\Psi, f\left(\Psi_{\text {coloop }}\right), f\left(\Psi_{\text {loop }}\right)\right)
$$

The previous statement explains how the relaxations of the complexes tell that the Tutte polynomial in classical matroid theory is actually an invariant to be attributed to the circuit and exchange axioms. The proofs of these theorems rely heavily on the consequences of the relaxations, i.e Theorems 3.4 3.5 and 3.6. 


\section{5 h-vectors and Stanley's conjecture}

Our starting point is the proof for rank 4 matroids in which a combinatorial approach to constructing a multicomplex was suggested. We begin with an $h$-vector decomposition that has a nice combinatorial interpretation. Given a complex $\Psi=(E, \Delta)$ and a set $A \subset E$, every face $F$ of $\Delta$ can be split in two pieces: $F \cap A$ and $F \backslash A$. We can partition the faces according to their part not in $A$. For any $\left.F \in \Delta\right|_{E-A}$, consider the order complex $\Psi_{A, F}:=\left(A, \Delta_{A, F}\right)=\left.(\Psi / F)\right|_{A}$. Here $\Delta_{A, F}=\left.\left(\operatorname{Link}_{\Delta} F\right)\right|_{A}$. Then the set of faces of $\Delta$ is the (disjoint) union of the faces of the $\Delta_{A, F}$ sets and writing this in terms of $h$-polynomials we get the following lemma.

Lemma 5.1 Given $A \subset E$, we have that

$$
h(\Delta, x)=\sum_{F \in \Delta !_{E \backslash A}}(1-x)^{(d-|F|)-r k \Delta_{A, F}} h\left(\Delta_{A, F}, x\right) .
$$

In particular, if $r k \Delta_{A, F}=d-|F|$ for every $\left.F \in \Delta\right|_{E \backslash A}$, we get that

$$
h(\Delta, x)=\sum_{\left.F \in \Delta\right|_{E \backslash A}} x^{|F|} h\left(\Delta_{A, F}, x\right) .
$$

This decomposition can be studied as related to shifted complexes.

Corollary 5.2 If $\Psi$ is a complex satisfying WEA or WIA, and $A$ is a subset of $E$ that contains $B_{0}$ such that $\left.\Psi\right|_{A}$ is pure, then the decomposition (8) of $h(\Delta, x)$ is satisfied.

We now relate the $h$-polynomial with internal activities and discuss a few facts about a poset analogue to the $<_{\text {int }}$ poset on the set of bases of an ordered matroid as defined in Las Vergnas (2001). From now on, all complexes $\Psi$ satisfy WEA and WIA.

Definition 5.3 The $<_{\text {int }}$ poset on the set $\mathcal{B}$ of basis is defined by $B_{1} \leq_{\text {int }} B_{2}$ if and only if IP( $\left.B_{1}\right) \subseteq$ $I P\left(B_{2}\right)$.

A further interesting class that contains ordered matroids and shifted complexes concerns the smallest lexicographic bases. In particular, recall that $B_{0}$ denotes the smallest lexicographic basis of $\Psi$ and $B_{v, 0}$ denotes the smallest lexicographic basis of $\Psi / v$.

Definition 5.4 An complex $\Psi=(E, \Delta)$ is said to satisfy the First Facet Property, abbreviated to FFP, if:
i. The rank of $\Psi=0$, or
ii. $\Psi$ has exactly one basis, or
iii. For every vertex $v$ of $\Psi$, the contraction $\Psi / v$ satisfies $F F P$ and $B_{v, 0} \subset B_{0}$.

It is a consequence of Lemma 2.4 in Dall (2015) that ordered matroids satisfy FFP and it is straightforward to verify the same statement for shifted complexes. The following theorem gives a combinatorial interpretation of Corollary 5.2 whenever the complex satisfies WEA, WIA and FFP.

Theorem 5.5 Assume that $\Psi$ satisfies WEA, WIA and FFP. Then for every basis $B$,

$$
I P(B)=F \cup I P\left(B \backslash F, \Psi_{B_{0}, F}\right) .
$$


Similar results were used in Klee and Samper (2015) to provide a combinatorial conjecture that implies Stanley's conjecture for matroids. We are now ready to present a relaxation of Stanley's conjecture. It is stated for a larger class of simplicial complexes and opens the door to various inductive techniques that are out of reach within matroid theory. We expect the technique of induction on the number of bases to have a variety of applications different from Stanley's conjecture.

Conjecture 5.6 There exists a map $\mathcal{F}$ from the class of ordered simplicial complexes satisfying WEA, WIA and FFP to the class of multicomplexes, such that the multicomplex $\mathcal{F}(\Psi)$ satisfies the following:

$i$. The set of variables appearing in $\mathcal{F}(\Psi)$ is $\left\{x_{i}: i\right.$ is a vertex of $\left.\left.\Delta\right|_{\left(E \backslash B_{0}\right)}\right\}$.

ii. The monomials of $\mathcal{F}(\Psi)$ are in bijection with bases of $\Psi$, in such a way that:

(a) for every basis $B$ the monomial associated under the bijection is denoted by $m_{B}$,

(b) the degree of $m_{B}$ equals to $|I P(B)|$,

(c) the support of $m_{B}$, i.e the set $\left\{e \in E: x_{e} \mid m_{B}\right\}$, is equal to $B \backslash B_{0}$.

iii. If $B_{1}<B_{2}$ in the in Gale $(\Psi)$ and $B_{1} \backslash B_{0} \subseteq B_{2} \backslash B_{0}$, then there is $B_{3}<B_{2}$ such that $\left|\operatorname{IP}\left(B_{2}\right)\right|=$ $\left|I P\left(B_{3}\right)\right|$ and $m_{B_{2}} \mid m_{B_{3}}$.

iv. The poset on $\mathcal{B}$ such that $B<B^{\prime}$ if and only if $m_{B} \mid m_{B^{\prime}}$ is an extension of $<_{i n t}$ and is extended by $\operatorname{Gale}(\Psi)$.

v. If $A \subseteq E$ contains $B_{0}$, then $\mathcal{F}\left(\left.\Psi\right|_{A}\right) \subseteq \mathcal{F}(\Psi)$.

We now provide a few results that aim to support the validity of the conjecture.

Theorem 5.7 Assume that the map $\mathcal{F}$ of Conjecture 5.6 exists for the class of all rank-d complexes satisfying WIA,WEA and FFP with $|E| \leq 2 d-1$. Then Conjecture 5.6 holds for rank-d complexes.

Since the Gale diagram of every matroid has a maximum, condition $i i i$. of Conjecture 5.6 implies Stanley's conjecture. In fact, this conjecture is a combinatorial version of the classification of $h$-vectors of Cohen-Macaulay complexes for the class of complexes satisfying WEA, WIA and FFP. It was originally proposed for matroids by Klee and Samper (2015), where it was shown for matroid of rank at most 4 . Internally perfect matroids defined by Dall (2015) satisfy the conjecture and are a potential candidate for a big class of matroids satisfying Conjecture 5.6

It is straightforward to see that every ordered complex whose facets are an order ideal of $\operatorname{Gale}(\Psi)$ satisfies WIA, WEA and FFP if $\Psi$ does. This opens the doors to do induction on the number of facets of the complex. This gives another piece of evidence and will hopefully serve as a source of inspiration for the general case.

Theorem 5.8 Conjecture 5.6 holds for shifted complexes.

Proof: Recall that a rank $d$ complex $\Psi=([n], \Delta)$ is shifted if and only if it is an order ideal of Young's lattice that is below the $d \times(n-d)$ box. A facet with vertices $v_{d}>v_{d-1}>\cdots>v_{1}$ corresponds to the partition $\lambda(B)=\lambda_{d} \geq \lambda_{d-1} \geq \cdots \geq \lambda_{1}$, where $\lambda_{i}=v_{i}-i$ (note that $\lambda_{i}$ might be equal to zero for some i). 
For every such partition $\lambda(B)$, where $B$ is a facet, we will construct a monomial $m_{\lambda}(d, n)$. We will drop $B, d, n$ from the notation when they are clear. Let us make some remarks first:

- $I A(B)=[r]$, where $r$ is the largest number such that $[r] \subseteq B$. This means that $|I P(B)|=\ell(\lambda)$, the length of $\lambda$. This gives the degree of $m_{\lambda}$.

- The set of variables of $m_{\lambda}$ are indexed by the set $B \backslash[d]$. The number of variables is the Durfee square, i.e the side length of the largest integer square fitting inside the Young diagram of $\lambda$.

This suggests that the construction should be inductive: the rows corresponding to the Durfee square of $\lambda$ should give the information of the support, and the rows below that should give the exponents of each variable. In what follows, we will present two different ways to construct the monomial $m_{\lambda}$ as a statistic of $\lambda$.

First we do it inductively. Notice that in small cases the construction is stratightforward (see the example below). Let $\operatorname{Dur}(\lambda)$ denote the partition obtained from $\lambda$ by removing the rows of the Durfee square. Assume that the side length of the Durfee square is $k$ and let $i_{1}<\cdots<i_{k}$ be vertices of $B$ corresponding to the first $k$ rows. Now $\operatorname{Dur}(\lambda)$ is a partition fitting in a $(d-k) \times k$, thus it has a monomial $x_{d-k+1}^{\alpha_{1}} x_{d-k+2}^{\alpha_{2}} \ldots x_{d}^{\alpha_{k}}$ of degree $\ell(\lambda)-k$ by induction (here some $\alpha_{i}$ may be zero). The monomial $m_{\lambda}$ associated to $\lambda$ is then $x_{i_{1}}^{\alpha_{1}+1} x_{i_{2}}^{\alpha_{2}+1} \ldots x_{i_{k}}^{\alpha_{k}+1}$.

There is a pictorial way to represent this in terms of the Young diagram, which we call the bouncing light construction. Imagine that the Young diagram has mirrors in the vertical boundaries. The left-hand side mirrors reflect lines parallel to the $x$-axis in the direction of the diagonal and the right hand side mirrors reflect lines coming in the direction of the diagonal to lines parallel to the $x$-axis. Now put a light on the right-hand side of each row of the Durfee square and shoot the light parallel to the $x$-axis. For each $i_{j}$ let $\beta_{j}$ be the number of times that the light bounces off the left wall and let $m_{\lambda}=x_{i_{1}}^{\beta_{1}} x_{i_{2}}^{\beta_{2}} \ldots x_{i_{k}}^{\beta_{k}}$. Then $m_{\lambda}$ is the desired monomial.

We end this section with two examples that illustrate the constructions.

Example 5.9 Consider the order ideal of the Young lattice fitting into a $3 \times 3$ box presented in Figure 2. Depicted in the figure are the Young diagrams. To the left of the diagram are listed the vertices of the corresponding facet with the variable vertices highlighted in red. Below the facets are the internally passive sets written in blue. To the right of the diagram is the corresponding monomial written in green.

Example 5.10 Consider the partition $(7,7,5,5,3,2,1)$. It fits into the $7 \times 7$ box. It corresponds to the facets $\{14,13,10,9,7,6,4,4\}$ in any complex it belongs to and the monomial associated is $x_{14} x_{13}^{2} x_{10} x_{9}^{3}$. The first four rows give the variables.

- The left hand side of the figure shows the inductive construction. The blue square is the Durfee and the top four rows give the answer. Remaining partition $(3,2,1)$ should be thought as fitting in $3 \times 4$ box, thus has a monomial in a subset of the variables $x_{5}, x_{6}, x_{7}, x_{8}$ and then we do an ordered substitution of variables: $x_{5} \mapsto x_{9}, x_{6} \mapsto x_{10}, x_{7} \mapsto x_{13}, x_{8} \mapsto x_{14}$.

- The right hand side gives the construction of the bouncing light. The variables $x_{14}, x_{13}, x_{10}, x_{9}$ correspond to colors red, blue, green and light blue respectively. 


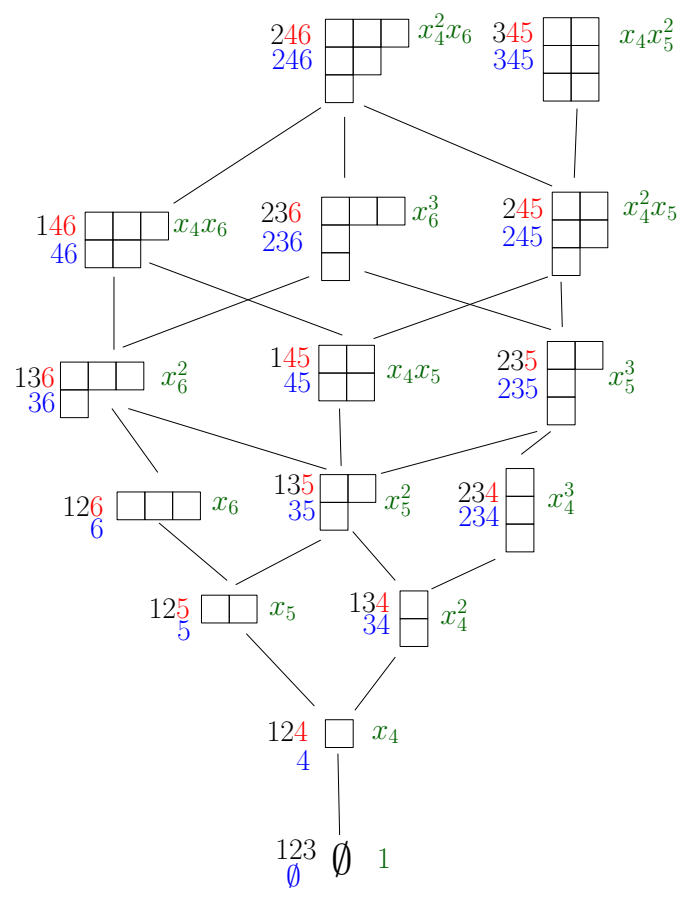

Fig. 1: An order ideal of the Young lattice

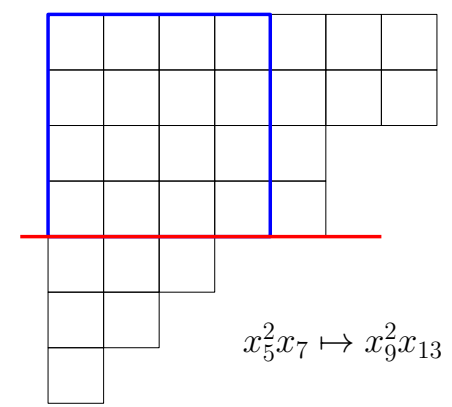

14

13

10

9

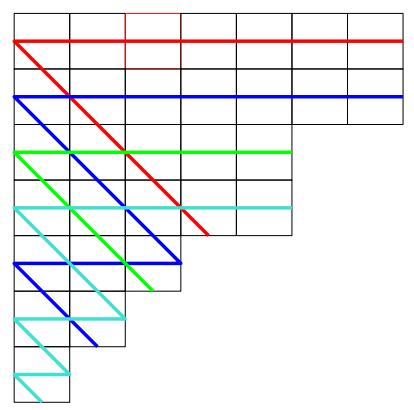

Fig. 2: Two ways to construct: inductively on the left and the bouncing light construction on the right. 


\section{Acknowledgements}

I would like to thank Isabella Novik, Steven Klee, Vic Reiner, Ernest Chong, Jeremy Martin and Gillaume Chapuy for several interesting conversations. I am also thankful with Hailun Zheng and Vasu Tewari for proofreading this manuscript and helping me improve the presentation.

\section{References}

A. Björner. The homology and shellability of matroids and geometric lattices. In Matroid applications, volume 40 of Encyclopedia Math. Appl., pages 226-283. Cambridge Univ. Press, Cambridge, 1992.

A. Constantinescu, T. Kahle, and M. Varbaro. Generic and special constructions of pure $O$-sequences. Bull. Lond. Math. Soc., 46(5):924-942, 2014. ISSN 0024-6093. doi: 10.1112/blms/bdu047. URL http://dx.doi.org/10.1112/blms/bdu047.

A. Dall. Internally Perfect Matroids, 2015. URL http://arxiv.org/abs/1510.04532.

J. A. De Loera, Y. Kemper, and S. Klee. $h$-vectors of small matroid complexes. Electron. J. Combin., 19 (1):Paper 14, 11, 2012. ISSN 1077-8926.

A. M. Duval. A common recursion for Laplacians of matroids and shifted simplicial complexes. Doc. Math., 10:583-618, 2005. ISSN 1431-0635.

A. M. Duval. The surprising similarity of shifted complexes and matroids, 2014. Slides available at http://www.math.utep.edu/Faculty/duval/papers/stanley70talk.pdf.

A. M. Duval and V. Reiner. Shifted simplicial complexes are Laplacian integral. Trans. Amer. Math. Soc., 354(11):4313-4344 (electronic), 2002. ISSN 0002-9947. doi: 10.1090/S0002-9947-02-03082-9. URL http://dx.doi.org/10.1090/S0002-9947-02-03082-9.

I. M. Gel'fand, R. M. Goresky, R. D. MacPherson, and V. V. Serganova. Combinatorial geometries, convex polyhedra, and Schubert cells. Adv. in Math., 63(3):301-316, 1987. ISSN 0001-8708. doi: 10.1016/ 0001-8708(87)90059-4. URL http://dx.doi.org.offcampus.lib.washington.edu/ 10.1016/0001-8708(87)90059-4.

S. Klee and J. A. Samper. Lexicographic shellability, matroids, and pure order ideals. Adv. in Appl. Math., 67:1-19, 2015. ISSN 0196-8858. doi: 10.1016/j.aam.2015.03.003. URL http://dx.doi.org/ $10.1016 / j . a a m .2015 .03 .003$.

C. Klivans. Combinatorial properties of Shifted Complexes, 2003. PhD Thesis, MIT.

W. Kook. Recurrence relations for the spectrum polynomial of a matroid. Discrete Appl. Math., 143(1-3): 312-317, 2004. ISSN 0166-218X. doi: 10.1016/j.dam.2003.10.008. URL http://dx.doi.org/ $10.1016 / \mathrm{j} . \mathrm{dam} .2003 .10 .008$.

W. Kook, V. Reiner, and D. Stanton. Combinatorial Laplacians of matroid complexes. J. Amer. Math. Soc., 13(1):129-148, 2000. ISSN 0894-0347. doi: 10.1090/S0894-0347-99-00316-1. URL http: //dx.doi.org/10.1090/s0894-0347-99-00316-1. 
M. Las Vergnas. Active orders for matroid bases. European J. Combin., 22(5):709-721, 2001. ISSN 01956698. doi: 10.1006/eujc.2001.0491. URL/http://dx.doi.org/10.1006/eujc.2001.0491. Combinatorial geometries (Luminy, 1999).

C. Merino. The chip firing game and matroid complexes. In Discrete models: combinatorics, computation, and geometry (Paris, 2001), Discrete Math. Theor. Comput. Sci. Proc., AA, pages 245-255 (electronic). Maison Inform. Math. Discrèt. (MIMD), Paris, 2001.

C. Merino, S. D. Noble, M. Ramírez-Ibáñez, and R. Villarroel-Flores. On the structure of the $h$-vector of a paving matroid. European J. Combin., 33(8):1787-1799, 2012. ISSN 0195-6698. doi: 10.1016/j. ejc.2012.04.002. URL http://0-dx.doi.org.opac.sfsu.edu/10.1016/j.ejc.2012. 04.002 .

S. Oh. Combinatorics related to the Totally Nonnegative Grassmannian, 2011. PhD Thesis, MIT.

S. Oh. Generalized permutohedra, $h$-vectors of cotransversal matroids and pure $O$-sequences. Electron. J. Combin., 20(3):Paper 14, 14, 2013. ISSN 1077-8926.

V. Reiner. Recent progress in the topology of simplicial complexes, 2001. Slides available at http://www.math.umn.edu/ reiner/Talks/transparencies.html.

J. A. Samper. Relaxing matroid axioms I: Independence, exchange and circuits. In preparation, 2015.

R. P. Stanley. Cohen-Macaulay complexes. In Higher combinatorics (Proc. NATO Advanced Study Inst., Berlin, 1976), pages 51-62. NATO Adv. Study Inst. Ser., Ser. C: Math. and Phys. Sci., 31. Reidel, Dordrecht, 1977. 